\title{
Nondestructive Quantification of Pharmaceutical Tablet Coatings using Terahertz Pulsed Imaging and Optical Coherence Tomography
}

\author{
Shuncong Zhong, ${ }^{1, a}$ Yao-Chun Shen, ${ }^{1, *}$ Louise Ho, ${ }^{2,3, \text { b }}$. May, ${ }^{4}$ J. Axel Zeitler, ${ }^{4}$ \\ Mike Evans, ${ }^{3}$ Philip F. Taday, ${ }^{3}$ Michael Pepper, ${ }^{3}$ Thomas Rades, ${ }^{2}$ Keith C. Gordon, ${ }^{5}$ \\ Ronny Müller, ${ }^{6}$ and Peter Kleinebudde ${ }^{6}$
}

${ }^{1}$ Department of Electrical Engineering and Electronics, University of Liverpool, L69 3GJ, UK

${ }^{2}$ School of Pharmacy, University of Otago, P.O. Box 56, Dunedin, New Zealand

${ }^{3}$ TeraView, Ltd., St. John’s Innovation Park, Cambridge CB4 0WS, UK

${ }^{4}$ Department of Chemical Engineering and Biotechnology, University of Cambridge, CB2 3RA, UK

5 MacDiarmid Institute for Advanced Materials and Nanotechnology, Department of Chemistry, University of Otago, P.O Box 56, Dunedin, New Zealand

${ }^{6}$ Institute of Pharmaceutics and Biopharmaceutics, Heinrich-Heine-University, D-40225, Germany

a: Present address: School of Mechanical Engineering and Automation, Fuzhou University, Fuzhou 350108, P.R. China

b Present address: Drug Delivery, Disposition and Dynamics, Monash Institute of Pharmaceutical Sciences, Monash University, 381 Royal Parade, Parkville, Vic. 3052, Australia

Abstract: Optical Coherence Tomography (OCT) and Terahertz pulsed imaging (TPI) are two powerful techniques allowing high quality cross-sectional images from within scattering media to be obtained non-destructively. In this paper, we report experimental results of using OCT and TPI for quantitatively characterizing pharmaceutical tablet coatings in the thickness range of $10-140 \mu \mathrm{m}$. We found that the spectral OCT system developed in-house has an axial resolution of $0.9 \mu \mathrm{m}$, and is capable of quantifying very thin coatings in the range of $10-60 \mu \mathrm{m}$. The upper limit of $60 \mu \mathrm{m}$ within the tablet coating and core is owing to the strong scattering of OCT light, which has relatively short wavelengths in the range of $0.5-$ $1.0 \mu \mathrm{m}$. On the other hand, TPI utilizes terahertz radiation which has substantially longer * Corresponding author. Tel: +44 (0)151 794 4575; fax: +44 (0)151 7944540.

E-mail address: y.c.shen@liv.ac.uk (Dr. Yaochun Shen) 
wavelengths in the range of hundreds microns, thus is less prone to the scattering problem. Consequently TPI has been demonstrated to be able to quantify thicker coatings in the range of $40-140 \mu \mathrm{m}$ and beyond. We concluded that OCT and TPI are two complementary analytical techniques for non-destructive and quantitative characterizing pharmaceutical tablet coatings.

Key words: Nondestructive testing; Optical Coherence Tomography; Terahertz Pulsed Imaging; Tablet coating thickness.

\section{Introduction}

Pharmaceutical tablet coating is one of the preferred means to control the release of active pharmaceutical ingredients (API) molecules in the human body [1]. For example, enteric coating is used to protect the API against degradation in the stomach and sustained-release coating is used to obtain an optimum release profile, and hence a desirable API absorption rate [1]. Sustained release coatings act as a diffusion barrier during drug release. Typically insoluble polymers are used for this purpose and depending on the polymer film thickness and porosity the drug release kinetics is controlled in such a dosage form. The quality of tablet coating will thus have direct implications on product performance. Lack of quality resulting in dose failures such as dose dumping may precipitate legal and commercial consequences for the manufacturer. It is hence of great interest to characterize coating properties such as coating thickness and coating uniformity for the purpose of quality control and quality assurance. A number of methods have been evaluated as means for coating quality assessment and monitoring. These methods range from coating weight gain to various destructive and non-destructive techniques [2, 3]. Routinely in the pharmaceutical industry, tablet coating is controlled by employing calculations on tablet weight gain during the coating processes with respect to the amount of coating solution applied. However, weight gain determination only provides the averaged value for a batch of tablets, and it does not give information on coating 
thickness and its uniformity of each individual tablet [4]. Terahertz pulsed imaging (TPI) has recently been demonstrated as a powerful technique for quantitatively characterizing coatings of individual pharmaceutical tablets $[5,6,7]$. In particular, terahertz radiation can penetrate most pharmaceutical excipients, and this makes TPI an increasingly popular tool for assessing information from various pharmaceutical solid dosage forms. These include coating thickness mapping, coating interface evaluation and tablet dissolution prediction [8]. However, the thinnest coating that could be precisely quantified is about $40 \mu \mathrm{m}$ for most common pharmaceutical coatings, using the state-of-the-art TPI system [7, 8]. Whilst this is sufficient for charactering many finished tablet coatings, a better detection limit is highly desirable for the quality assessment of very thin tablet coatings, and for online monitoring of coating process where such thin coatings (corresponding to the early stage of the coating process) is of particular interest.

Optical coherence tomography (OCT) is also a noninvasive and cross-sectional imaging technique which permits, for example, three-dimensional images with micrometer-resolution to be obtained from within the retina [9-10]. However, most commercially available OCT systems are developed and optimized for medical applications. To date, applications of OCT beyond biomedicine have been scarce. Nevertheless, there have been some reports on using OCT for non-destructive evaluation of thickness of papers [11], and surface roughness, gloss and bulk properties of flat-faced tablets [12]. Very recently, OCT has also been evaluated for characterizing coating thickness of pharmaceutical tablets [13] and for imaging pharmaceutical tablets [14].

In this paper, we report an experimental investigation of using OCT and TPI for quantitative characterization of pharmaceutical tablet coatings in the thickness range of $10-$ $140 \mu \mathrm{m}$. The same set of pharmaceutical tablets was measured using both a in house OCT system and a commercial TPI system. We found that OCT is better suited for analysis of 
thinner coatings owing to its higher axial resolution whilst TPI is less prone to scattering problems thus is well positioned for characterizing pharmaceutical tablets with thicker coatings.

\section{Methods and Materials}

For all TPI measurements reported here the TPI imaga2000 system (TeraView Ltd, Cambridge, UK) was used and operated at a rapid scan mode, i.e., at an acquisition rate of 30 complete waveforms per second. At each measurement point, the terahertz radiation reflected from a tablet sample was recorded as a function of time over a scan range of $2 \mathrm{~mm}$ optical delay. The TPI imaga 2000 system is specifically developed for the fully automated scan of typical pharmaceutical solid dosage forms that usually have curved surfaces. A six-axis robot system was employed to handle the tablets. This ensures that the tablet is always at the terahertz focus position with its surface perpendicular to the terahertz probe during a TPI measurement [7]. Note that the terahertz radiation used here is broadband, covering a spectral range of $5-100 \mathrm{~cm}^{-1}(0.15-3 \mathrm{THz})$. The spot size of the focused terahertz beam at the tablet surface is estimated to be about $200 \mu \mathrm{m}$ in diameter at its centre frequency of $1.5 \mathrm{THz}\left(50 \mathrm{~cm}^{-}\right.$ $1)$.

All OCT measurements were carried out using a in house OCT system. As shown in Fig. 1, the light from a $50 \mathrm{~W}$ halogen lamp was delivered onto the entrance pupil of the interferometer using a multimode fiber. The fiber end was then imaged at a ratio of 1:1 onto the surface of the sample and the reference, using a biconvex lens through a beam-splitter (50:50). The light that was reflected/scattered back from both reference and sample was collected using another biconvex lens and then imaged back into the entrance slit of a spectrometer. Interference will occur when both the "sample image" and the "reference image" are spatially matched in size and orientation within the corresponding transverse coherence distance, and their path lengths are matched within the coherence length of the 
light source. A PZT actuator was introduced to shift the phase of the reference beam, which allows the phase-shifting method [12] to be used to increase the measurement sensitivity. One distinct feature of our OCT system is the combination of a broadband CCD-based spectrometer with a white light source of an extremely short coherence length. This configuration provides good signal-to-noise ratio and at the same time excellent axial resolution. The multimode fiber used was chosen to have a diameter of $200 \mu \mathrm{m}$, to make sure that the OCT light beam and the TPI probe beam have a comparable spot size at the sample surface.

The sample used in the present work was a batch of pharmaceutical tablets with a single coating layer. Tablet cores were biconvex (3 $\mathrm{mm}$ in height, $8 \mathrm{~mm}$ in diameter, average weight of $252 \mathrm{mg}$ ), and contained 10\% w/w diprophyllin (API), 84.5\% w/w lactose monohydrate (Flowlac ${ }^{\circledR}$ 100), 5\% w/w vinylpyrrolidone-vinyl acetate copolymer (Kollidon ${ }^{\circledR}$ V64) and $0.5 \% \mathrm{w} / \mathrm{w}$ magnesium stearate. The coating formulation used is as follows: $50 \% \mathrm{w} / \mathrm{w}$ polyvinyl acetate (Kollicoat ${ }^{\circledR}$ SR 30D), 6\% w/w polyvinyl alcoholpolyethyleneglycol graft coplmer (Kolicoat ${ }^{\circledR}$ IR), 0.075\% w/w polyoxyethylene(20) sorbitan monooleate (Polysorbate 80 ), $0.3 \% \mathrm{w} / \mathrm{w}$ glycerolmonostearate, $0.75 \% \mathrm{w} / \mathrm{w}$ triethylcitrate and $42.87 \%$ w/w deionised water. The tablets were randomly selected after the following amounts of sustained-release polymer were applied: 1.8, 3.6, 5.5, 7.3, 9.1, 10.9, 12.7, 14.5 and 18.2 $\mathrm{mg} / \mathrm{cm}^{2}[16]$

\section{Results and Discussions}

In order to characterize the performance of the in-house OCT system, we firstly measured a layered sample of polymer film with known thickness. Fig. 2 (a) shows the broadband OCT spectral interferogram obtained for the layered sample (curve A), whilst the curve B and C are the corresponding spectrum of the sample and the reference arm, respectively.. The 
measured spectrum of the reference arm (curve C of Fig.2 (a)), which represents the overall spectral range of the instrument including the light source, has a centre wavelength of $700 \mathrm{~nm}$ and a full-width-at-half-maximum (FWHM) of $236 \mathrm{~nm}$. The OCT axial resolution $\Delta z$ is given by $\Delta z=2 \ln 2 \lambda_{0}^{2} / \pi \Delta \lambda$, where $\lambda_{0}$ is the centre wavelength and $\Delta \lambda$ is the FWHM of the light source. The achieved axial resolution obtained from FWHM is about $0.9 \mu \mathrm{m}$ in air (Fig. 2(d)), which is amongst the best ever reported [13]. Fig. 2 (b) shows two spectral interferograms obtained with a phase difference of $\pi$, using the phase-shifting method [12]. The corresponding differential OCT interferogram (the difference of these two interferograms) is shown in Fig.2 (c). This has greatly improved the signal-to-noise ratio and the measurement sensitivity. Consequently both coating layers of the sample can be resolved from its corresponding OCT-FFT waveform shown in Fig. 2(d). The thickness of the first and the second layer were determined to be 6 and $18 \mu \mathrm{m}$, respectively. Note that the third peak (at $\mathrm{z}=24 \mu \mathrm{m}$, as indicated on Fig.2 (d)) has an amplitude of $0.027 \mathrm{~dB}$. This feature is very small but is still well above the background noise level (0.0005 dB) confirming that our OCT system has extremely good signal-to-noise ratio thus good measurement sensitivity.

The axial resolution of the OCT setup was determined to be $0.9 \mu \mathrm{m}$ with a signal-to-noise ratio of 2000. A set of tablets with a film coating weight gain ranging from $1.8 \mathrm{mg} / \mathrm{cm}^{2}$ to 7.2 $\mathrm{mg} / \mathrm{cm}^{2}$ was measured using the OCT spectrometer. Fig. 3(a) shows a typical OCT-FFT waveform recorded for a tablet with a weight gain of $5.4 \mathrm{mg} / \mathrm{cm}^{2}$. From the OCT-FFT waveform, the tablet core roughness, coating roughness and thickness can be obtained. Unlike the OCT-FFT waveform in Fig. 2(d) where a sharp reflection peak (FWHM $0.9 \mu \mathrm{m}$ ) from the sample surface is observed, the OCT-FFT waveforms in Fig. 3(a) show a broad surface reflection feature comprising many sharp sparks. This can be explained as follows: (1) as discussed in the previous section the diameter of the OCT light spot size at the sample surface was chosen to be similar to that of terahertz probe beam (about $200 \mu \mathrm{m}$ ) in order to make 
reasonable comparison of OCT and TPI results; (2) for a sample with a smooth surface, the surface reflection feature across the entire sampling area of $200 \mu \mathrm{m}$ diameter will appear at the same position $(\mathrm{z}=0 \mu \mathrm{m})$. This leads to the sharp peak in the OCT-FFT waveform as shown in Fig. 2(c); (3) for a rough surface, the surface height varies over the sampling spot surface. Therefore the feature reflected/scattered from different parts of the surface will appear at slightly different position $(-\Delta \mathrm{z}<\mathrm{z}<\Delta \mathrm{z}$, where $\Delta \mathrm{z}$ is the surface roughness). This results in a broad envelope comprising many sharp sparks with each spark corresponding to light reflected/scattered from the portion of the surface of the same height.

By fitting the measured OCT-FFT waveforms with a Gaussian function, we are able to quantify the coating thickness as the peak position difference between the two fitted curves (shown as the solid curve in Fig. 3(a)). Fig. 3(b) shows the OCT b-scan maps of the same tablet with a coating polymer weight gain of $9 \mathrm{mg} / \mathrm{cm}^{2}$, from which the mean thickness of this specific tablet was determined to be $52 \mu \mathrm{m}$. Note that the OCT system was also used to measure tablets with a weight gain above $9 \mathrm{mg} / \mathrm{cm}^{2}$. However, for these samples the OCT signal from the coating/core interface was too weak to be used for reliable coating thickness analysis. This weaker OCT signal obtained from the thicker coatings was most likely caused by the strong scattering of the OCT signal within the tablet coating for this specific set of coating/core materials.

After OCT analysis, the same set of tablets was analyzed using the TPI system. Fig.3(c) shows a typical terahertz waveform recorded for a coated tablet, with Fig. 3(d) showing the corresponding TPI b-scan map. Noted here that results shown in Fig.3 (a)-(d) were obtained using the same tablet with a weight gain of $5.4 \mathrm{mg} / \mathrm{cm}^{2}$. Again, the coating thickness was calculated as the peak position difference between surface reflection feature and the coating/core interface reflection feature. The wavelength of the terahertz radiation used for the TPI experiments is about three orders of magnitude larger than that of the OCT light. 
Therefore, scattering within the tablet coating, the major limiting factor in the OCT measurement, is no longer an issue in the TPI measurement. Consequently, terahertz radiation can penetrate deeper into the tablet sample and TPI is able to quantify thicker coating layers as compared to OCT. As shown in Fig. 4, TPI was successfully used for the quantitative characterization of tablet coatings of a weight gain of $7.3-18.2 \mathrm{mg} / \mathrm{cm}^{2}$ (thickness range of $40-140 \mu \mathrm{m})$. On the other hand, we also found that the thinnest coating layer that could be precisely quantified using TPI is about $40 \mu \mathrm{m}$ for this specific set of tablets, which is in good agreement with previous report $[7,16]$. TPI technology could not provide precise coating thickness information for tablets with a weight gain of $1.8,3.6$ and $5.5 \mathrm{mg} / \mathrm{cm}^{2}$. In contrast, OCT technology is capable of measuring such thin tablet coatings. As summarised in Fig. 4, a linear relationship $\left(\mathrm{R}^{2}=0.96\right)$ between the coating thickness and the amount of polymer applied was observed in the full weight range of $1.8-18.2 \mathrm{mg} / \mathrm{cm}^{2}$ (thickness range of 10 $120 \mu \mathrm{m}$ ) investigated using both techniques. Note that neither TPI nor OCT alone can provide coating thickness information across such a broad range of coating thickness.

\section{Concluding Remarks}

In conclusion, operating at shorter wavelength than TPI, OCT provides higher axial resolution which makes it a better choice to analyse thin coatings. Terahertz radiation has a much longer wavelength thus is less prone to scattering problems and hence TPI can be used to analyze thicker coatings as well as for imaging the internal structures deeper inside a tablet. The combination of OCT and TPI would provide the pharmaceutical industry with a complementary and powerful analytical technique for the quantitative characterization of pharmaceutical tablet coatings in a thickness range from a few microns to a few hundreds microns.

\section{Acknowledgement}


The authors would like to acknowledge financial support from UK Technology Strategy Board (AB293H). S. Zhong would like to thank Fuzhou University Research Fund, Scientific Research Foundation for the Returned Overseas Chinese Scholars, State Education Ministry, P. R. China, and National Natural Science Foundation of China (51005077).

\section{References}

[1] G.M. Jantzen and J.R. Robinson. Sustained and controlled-release drug delivery systems. Modern Pharmaceutics, 4th ed., G. S. Banker and C. T. Rhodes, Eds. (New York, Marcel Dekker. 2002; 501-528.

[2] J.A. Zeitler and L.F. Galdden. In-vitro tomography and non-destructive imaging at depth of pharmaceutical solid dosage forms. Eur J. Pharm. Biopharm. 2008; 71: 2-22.

[3] C. Cahyadi, A.D. Karande, L.W. Chan, and P.W.S. Heng. Comparative study of nondestructive methods to quantify thickness of tablet coatings. Int. J. Pharm. 2010; 398: 39-49. [4] J.D. Perez-Ramos, W.P. Findlay, G. Peck, and K.R. Morris. Quantitative analysis of film coating in a pan coater based on in-line sensor measurements. AAPS PharmSciTech 2005; 6(1): E127-E136 .

[5] A.J. Fitzgerald, B.E. Cole, and P.F. Taday. Nondestructive analysis of tablet coating thickness using terahertz pulsed imaging. J. Pharm. Sci. 2005; 94: 177-183.

[6] J.A. Zeitler, Y.C. Shen, C. Baker, P.F. Taday, M. Pepper, and T. Rades. Analysis of coating structures and interface in solid dosage forms by three dimensional terahertz pulsed imaging. J. Pharm. Sci. 2007; 96: 330-340. 
[7] Y.C. Shen and P.F. Taday. Development and Application of Terahertz Pulsed Imaging for Non-destructive Inspection of Pharmaceutical Tablet. J. Sel. Top. in Quantum Electron. 2008; 14: 407-415.

[8] L. Ho, R. Müller, M. Römer, K.C. Gordon, P. Kleinebudde, M. Pepper, T. Rades, Y.C. Shen, P.F. Taday, and J.A. Zeitler, "Applications of terahertz pulsed imaging to sustainedrelease tablet film coating quality assessment and dissolution performance. J. Control. Rel. 2008; 127: 79-87.

[9] L. Maurer and H. Leuenberger. Terahertz pulsed imaging and near infrared imaging to monitor the coating process of pharmaceutical tablets. Int. J. Pharm. 2009; 370: 8-16.

[10] R.A. Leitgeb, C.K. Hitenberger, and A.F.Fercher. Performance of Fouier domain vs. time domain optical coherence tomography. Opti. Express 2003; 11: 889-894.

[11] T. Prykari, J. Czajkowski, E. Alarousu and R. Myllyla. Optical coherence tomography as an accurate inspection and quality evaluation technique in paper industry. Optical Review 2010; 17: 218-222.

[12] M. Juuti, H. Tuononen, T. Prykari, V. Kontturi, M. Kuosmanen, E. Alarosusu, J. Ketolainen, R. Myllyla, and K. Peiponen. Optical and terahertz measurement techniques for flat-faced pharmaceutical tablets: a case study of gloss, surface roughness and bulk properties of starch acetate tablets. Meas. Sci. Technol. 2009; 20: 1-9.

[13] S. Zhong, H. Shen, Y.C. Shen, J.A. Zeitler, L. Ho, M. Evans, P.T. Taday, M. Pepper, T. Rades, K.C. Gordon, R. Muller and P. Kleinebudde, "Noninvasive 3D characterization of Layered samples using Terahertz pulsed imaging and infrared optical coherence tomography”, Proceedings of the IEEE international Conference on Infrared, Millimeter and Terahertz waves, 2009.

[14] J.M.A. Mauritz, R.S. Morrisby, R.S Hutton, C. H. Legge, and C.F. Kaminski. Imaging pharmaceutical tablets with optical coherence tomography. J. Pharm. Sci. (to be published). 
[15] Z.H Ma, R.K. Wang, F. Zhang, and J.Q. Yao. Spectral optical coherence tomography using two-phase shifting method. Chinese Phys. Lett. 2005; 22(8): 1909-1912.

[16] L. Ho, R. Müller, K.C. Gordon, P. Kleinebudde, M. Pepper, T. Rades, Y.C. Shen, P.F. Taday, and J.A. Zeitler. Terahertz pulsed imaging as an analytical tool for sustained-release tablet film coating. Eur. J. Pharm. Biopharm. 2009; 71: 117-123.

[17] Y.C. Shen, P.F. Taday, D.A. Newnham, M.C. Kemp, and M. Pepper. 3D chemical mapping using terahertz pulsed imaging, Terahertz and Gigahertz Electric and Photonics IV. Proc. SPIE 2005; 5727: 24-31.

[18] S. Saarakkala, S.Z. Wang, Y.P. Huang, and Y.P. Zheng. Quantification of the optical surface reflection and surface roughness of particular cartilage using optical coherence tomography. Phys. Med. Biol. 2009; 54: 6837-6952. 


\section{Captions:}

Fig. 1 Schematic diagram of the spectral-domain OCT experimental set-up. BS: 50:50 beamsplitter; PZT: piezoelectric transducer.

Fig. 2 (a) Extremely broadband OCT spectral interferogram (curve A) obtained by CCDbased interferometer. Curve B and C are the corresponding spectrum measured for the sample and the reference arms respectively; (b) Two OCT spectral interferograms obtained with a phase difference of $\pi$, using the phase-shifting method; (c) The differential OCT spectral interferogram obtained after applying the phase-shift method; and its corresponding OCTFFT waveform (c). The axial resolution, defined as the FWHF of the main peak of the FFTOCT waveform, was determined to be $0.9 \mu \mathrm{m}$. The inset of Fig. 2 (d) shows the schematic diagram of a layered sample which is a plastic plate with a 6- $\mu$ m-thick cling film layer and a 18- $\mu$ m-thick polymer layer.

Fig.3 OCT-FFT waveform (a) and OCT cross-section map (b) recorded for a tablet with a weight gain of $5.4 \mathrm{mg} / \mathrm{cm}^{2}$. The solid lines in (a) are the best fitting curves using Gaussian function, and the coating thickness was determined to be $52 \mu \mathrm{m}$. The TPI terahertz waveform (c) and TPI b-scan map (d) were recorded for the same tablet. Note that TPI measures the electric field of the reflected terahertz radiation and the negative minimum peak at about 52 $\mu \mathrm{m}$ indicates that the refractive index of the tablet core is larger than that of the tablet coating. Noted here that results shown in Fig.3 (a)-(d) were obtained using the same tablet with a weight gain of $5.4 \mathrm{mg} / \mathrm{cm}^{2}$.

Fig.4 Correlations between tablet coating thickness and the amount of polymer applied. The solid diamonds are TPI results whilst the open circles are OCT results; The inset on the right- 
hand side shows TPI coating thickness maps obtained for tablets with the same weight gain of $18.2 \mathrm{mg} / \mathrm{cm}^{2}$. There is large tablet-to-tablet variation in the coating thickness for the tablets with the same weight gain. 


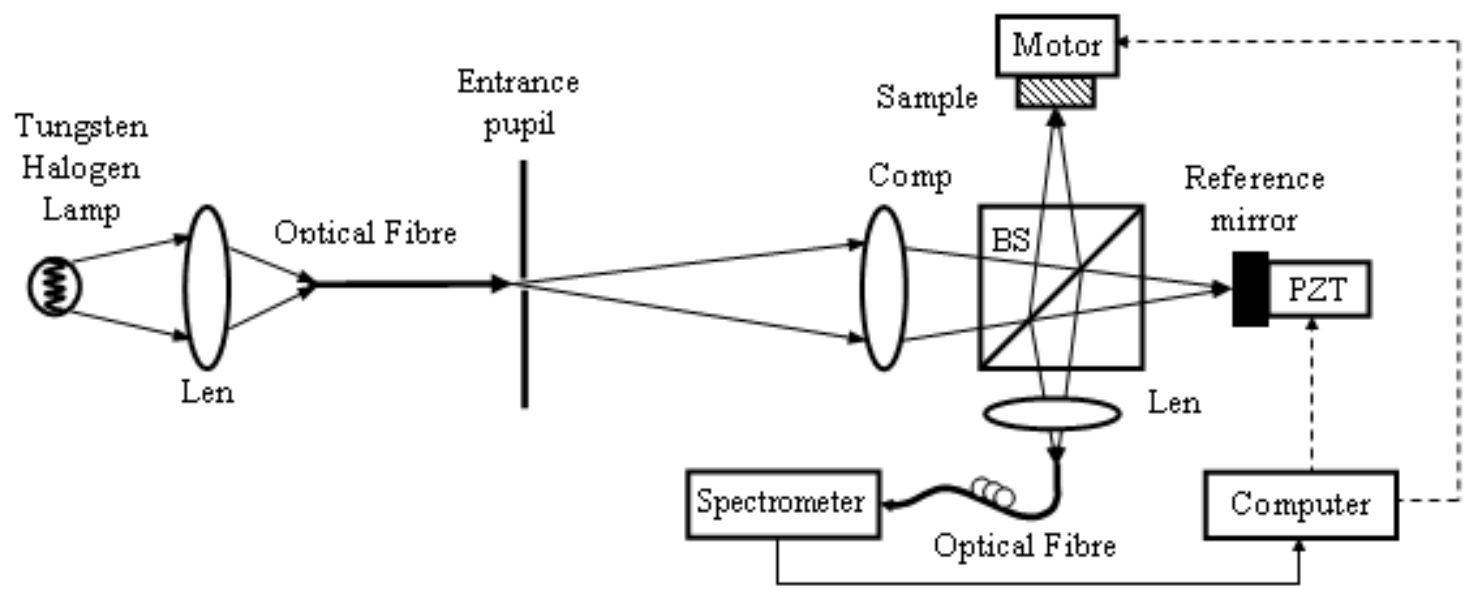

Fig.1
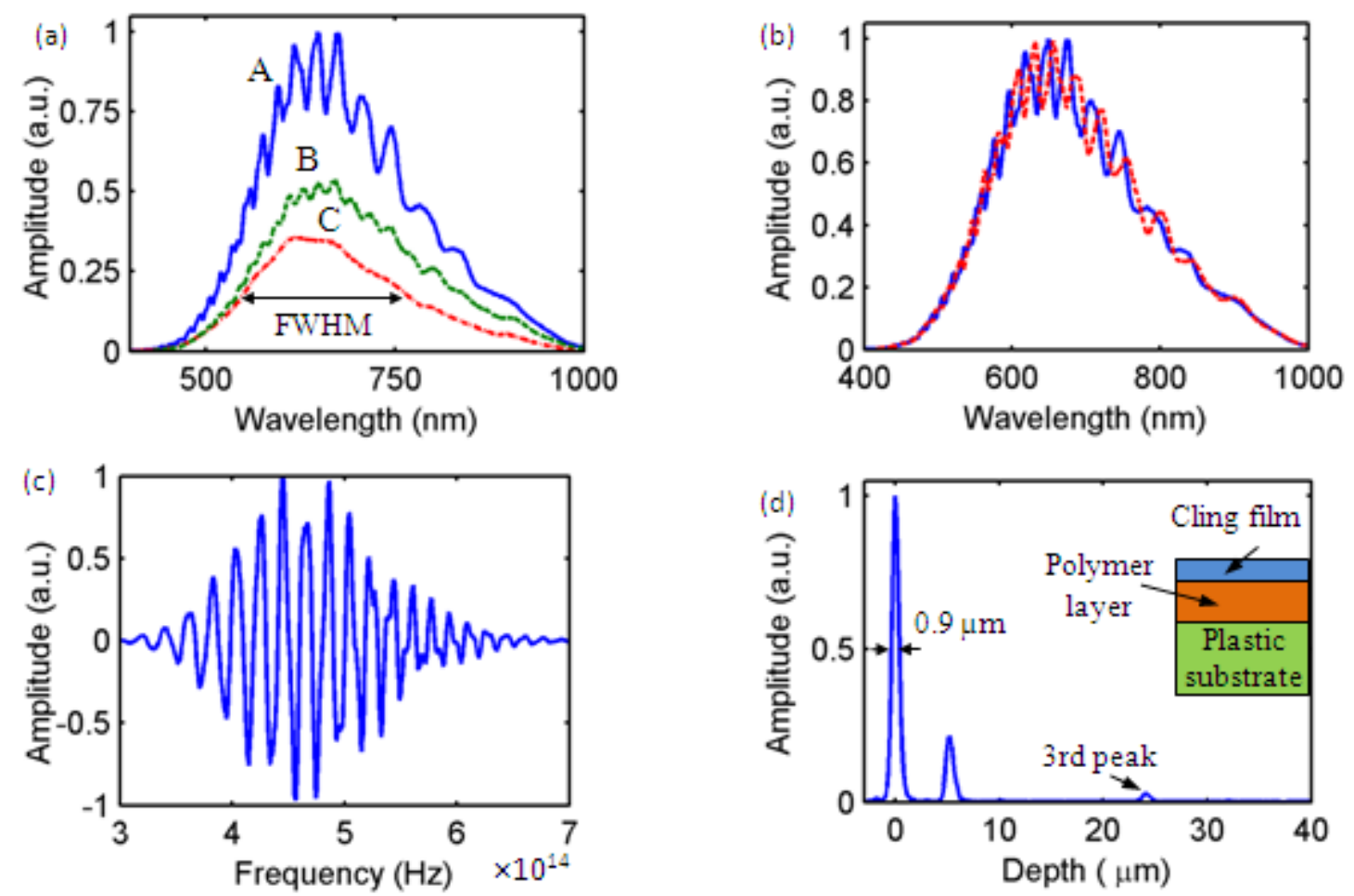

Fig.2 

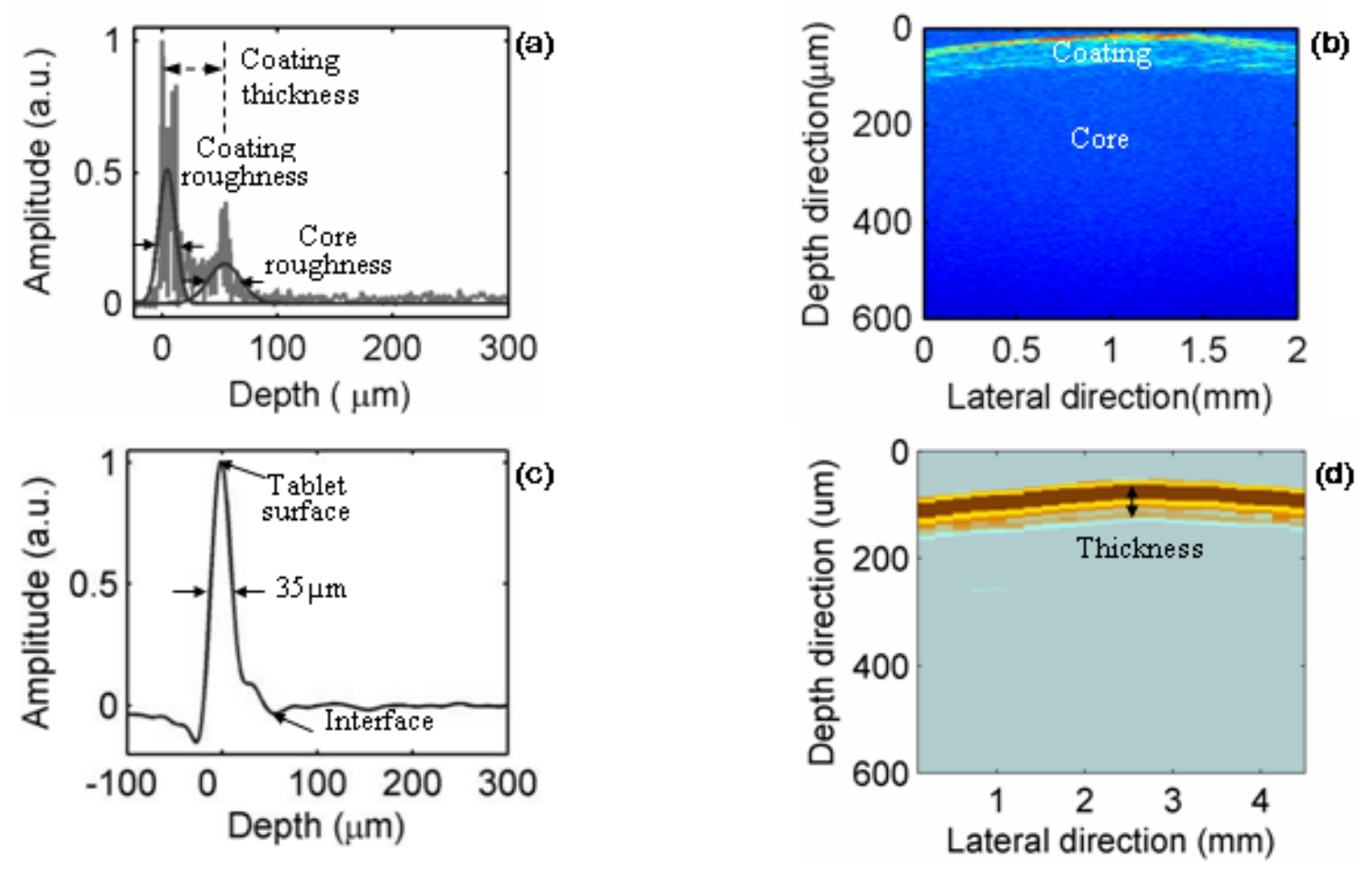

Fig.3 

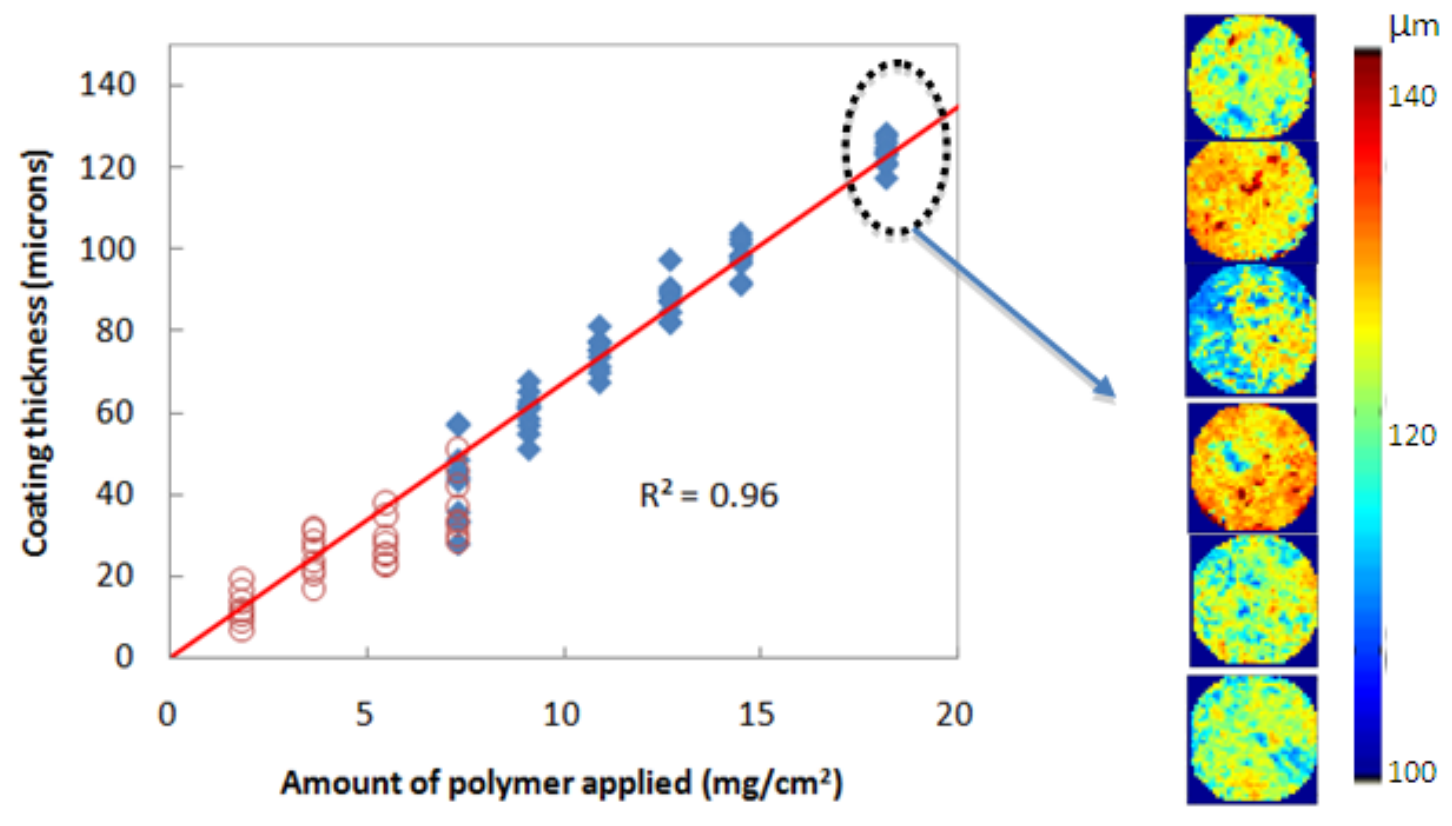

Fig.4 